A geometric formulation of the Law of Aboav - Weaire in two and three dimensions

J. K. Mason, R. Ehrenborg, E. A. Lazar

November 7, 2011

Journal of Physics A: Mathematical and Theoretical 
This document was prepared as an account of work sponsored by an agency of the United States government. Neither the United States government nor Lawrence Livermore National Security, LLC, nor any of their employees makes any warranty, expressed or implied, or assumes any legal liability or responsibility for the accuracy, completeness, or usefulness of any information, apparatus, product, or process disclosed, or represents that its use would not infringe privately owned rights. Reference herein to any specific commercial product, process, or service by trade name, trademark, manufacturer, or otherwise does not necessarily constitute or imply its endorsement, recommendation, or favoring by the United States government or Lawrence Livermore National Security, LLC. The views and opinions of authors expressed herein do not necessarily state or reflect those of the United States government or Lawrence Livermore National Security, LLC, and shall not be used for advertising or product endorsement purposes. 


\title{
RESPONSE FOR: A GEOMETRIC FORMULATION OF THE LAW OF ABOAV-WEAIRE IN TWO AND THREE DIMENSIONS
}

\author{
J. K. MASON, R. EHRENBORG AND E. A. LAZAR
}

\section{REFEREE ONE}

We would like to begin by expressing our sincere gratitude to this referee for carefully reading the manuscript and for providing numerous recommendations that have, in our opinion, significantly strengthened the paper. We have attempted to carefully implement all of this referee's suggestions, and would certainly welcome any further comments.

(1) This is certainly a valuable recommendation of the referee, namely, to clarify our explanation of the average excess curvature. The brief sentence quoted by the referee in this comment is changed in the current manuscript to read "Notice that by Lemma 1, the quantity on the left is the expectation value of the sum of the integrals of the curvature over the boundaries of the $n+1$ grains in any given $S_{i}$. Meanwhile, by the same lemma, the expression $2 \pi \cdot(n+1)-\pi / 3 \cdot\langle n\rangle \cdot(n+1)$ is the expectation value of the sum of the integrals of the curvature over the boundaries of $n+1$ grains, chosen independently and randomly from the entire population in $\Omega$. This means that $\epsilon_{n}$ is the difference of the expectation values of the integrated curvature over the boundaries of two populations of grains, one given by a cluster around a central grain with $n$ neighbors, and the other by the same number of grains chosen randomly from the cell complex." While this does not differ from the referee's interpretation of the original text, we hope that this will be much less ambiguous.

(2) The referee is absolutely correct that certain values of the $c_{k}$ would result in the expansion being finite for all large positive values of $k$, and has our sincere gratitude for pointing this out to us. While reflecting further on this issue, we realized that there is no need to work with a Laurent series after all. Since our concern is with the expansion of a function over the reals, a Taylor series would do perfectly well. The possible singularity at $n=0$ is avoided by performing the expansion around some other point, e.g. the average value of $n$. This is reflected in numerous changes in our development from equation 36 to equation 39 of the present manuscript. Of particular note is that the mathematical difficulty pointed out by the referee is removed, the general expression for $g_{n}$ in equation 38 is simplified, the suggested practical expression for $g_{n}$ in equation 39 is slightly more complicated, and figures 4-7 are completely redone. The text in this portion of the manuscript has been modified as well to accommodate these changes.

(3) We thank the referee for pointing out this typo, and have corrected the final sentence of the conclusion. 
(4) We apologize for this omission. As far as we can recall, the supplementary material was submitted along with the article, though apparently not included in automatically generated manuscript. We will try to communicate with the editors to ensure that this material is included with the manuscript in the future.

(5) The article in question has been accepted for publication since the original submission of this manuscript, and we are glad to include the complete citation information. Meanwhile, the sentence "...two- and three-dimensional versions of a simulated microstructure evolved into the steady-state regime by the algorithms of Lazar [1,2]" in the second paragraph of section 5 has been expanded to read "Our simulations of normal grain-growth move a discretized grain boundary in accordance with local versions of the von Neumann-Mullins relation [3, 4] and the MacPherson-Srolovitz relation [5] in two and three dimensions, respectively. At the point that the statistical features of the microstructure no longer measurably change with continued grain coarsening, the cell complex is regarded as being in the steady-state. Further details of the implementation of this algorithm are provided by Lazar [1, 2]." We would be perfectly willing to provide more information, should the referee feel that to be warranted.

(6) As requested by the referee, the values of the $c_{k}$ used in the plots in figures 4-7 are now included in the captions below the figures, with the number of significant digits reported indicating the uncertainties in the parameter values. We have also included the value of $\mu_{2}$, the second central moment of the distribution of the number of sides, in the caption since this is necessary to reproduce the figures.

\section{REFEREE TwO}

We would like to thank this referee for his comments, and welcome the opportunity that this affords to discuss the intent and reach of this paper in more detail.

(1) We absolutely agree with the referee that one of the central difficulties with research into the Law of Aboav-Weaire is identifying the relation that is being investigated, if not with the claim that this places our paper on quicksand. From our review of the available literature, the prevailing understanding is that the Law of Aboav-Weaire comprises roughly three distinct statements:

(a) For many two-dimensional cell complexes, grains with a small number of sides tend to be surrounded by grains with a large number of sides, and vice versa. This statement is given in the first sentence of our introduction, and appears to be generally agreed upon in all available literature on the subject.

(b) For many two-dimensional cell complexes, the average number of sides of a grain neighboring a grain with $n$ sides is reasonably well described by $5+\left(6+\mu_{2}\right) / n$. This statement is given in the second sentence of our introduction. While the expression given by Weaire generally performs well, many researchers have proposed other expressions in attempts to reduce the observed error, though they are for the most part similar to Weaire's expression. 
(c) The cell complexes that follow the Law of Aboav-Weaire is the set of natural cell complexes. This part of the usual understanding of the Law of Aboav-Weaire is given in the third paragraph of the introduction.

Statements (a) and (b) are essentially empirical in nature. We provide several references from a much larger body of supporting literature in the final sentence of the first paragraph of the introduction. Our intention with giving these as the first and second sentences of the introduction was to make them absolutely clear to the reader, though we would be open to suggestions for other means to present them. Meanwhile, the third statement is not nearly as well-founded as the other two. The central difficulty is with defining the features that make a cell complex natural, apart from the usual meaning of naturally occurring. Indeed, the referee seems to be in agreement with us on this point, per the referee's second comment and our fifth paragraph of the introduction.

With regard to the specifics of the referee's first comment, the Law of AboavWeaire does seem to be simple to state. However, this does not occur in the literature as a single precise mathematical expression, but rather as the collection of observations and suppositions given above. The introduction to our paper does seem to be the correct place to outline the prevailing understanding of the Law of Aboav-Weaire, with all of the accompanying ambiguities and exceptions. We do not feel that we may be held accountable for this historical state of affairs merely because we explain the situation though. Rather, the sentiment of the referee seems to be in line with our own, that there is a need to formulate a precise mathematical statement that roughly encompasses the statements given above. This is exactly the subject of our paper, though arriving at the resulting statements requires considerable development and would likely only become clear to the reader who proceeded as far as section 4 or section 5 in our original document. As an emphasis that this is our intention, our central result is now presented as the second unlabeled equation in the introduction.

(2) The answer to the referee's question is that nearly all researchers and papers on the subject regard the expressions proposed by Weaire [6] or Aboav [7] as the mathematical content of the Law of Aboav-Weaire, as the name suggests. Our equation 38 , the same as the second unlabeled equation in the introduction, simplifies to these expressions for certain conditions, as is mentioned in the fifth paragraph of the introduction and in the text following equation 38. The difference is that our derivation of equation 38 does not rely on empirical observations or heuristic arguments, but rather on purely geometric considerations. However, the referee's principle concern with this comment seems to be that in the absence of a specific cell complex, the function $g_{n}$ cannot be given in closed form. This is certainly the case, though we point out that our equation 38 is not restricted to any particular cell complex and is not a closed form expression.

(3) Our assertion that we "place [the Law of Aboav-Weaire] on a more solid foundation" occurs in the abstract, where the following sentence describes more specifically the meaning of our statement. That is, "we derive exact local forms of the Law of AboavWeaire, and an exact global form that is identical in two and three dimensions except for a single parameter $\zeta$ ". We regret that there is limited space in the abstract 
to expand more on the details presented in the paper, and hope that the referee understands that the space required to adequately elaborate on the specifics must be relegated to the body of the manuscript.

(4) The referee is of course correct that the Law of Aboav-Weaire is known to not hold for a Poisson-Voronoi structure. Our manuscript already includes several references that make this point in the second sentence of the fifth paragraph of the introduction. The usual explanation for this departure from the Law of Aboav-Weaire is that the Poisson-Vornoi structure is not natural, that is, does not frequently occur in nature where processes including the minimization of boundary energy influence the statistics of the cell complex. We would like to point out to the referee that our development does apply to the Poisson-Voronoi structure though, as figure 6 indicates. Indeed, the variations in the coefficients of equation 38 that occur in section 5 provide the possibility of identifying what makes a structure natural or otherwise, and should with further development make clear the class of models to which the Law of Aboav-Weaire applies. This relates directly to the final sentence of this comment of the referee, for which we are grateful as a suggestion for further research.

The referee will find, on a more thorough review of our manuscript, that there is no mention of a mean-field type approximation anywhere in our document. We do not know how to make our intention in this regard any more clear than by explicitly stating in the introduction that we do not make a mean-field approximation of this kind, though such a statement would seem out of place outside the context of the current discussion.

(5) We apologize for the apparent confusion surrounding the final paragraph of the introduction. Please allow us to clarify our statement that a 'cell complex' should be regarded as a regular CW complex. While the complete definition of a regular CW complex is considerably more involved, the explanation below should suffice. Let an $n$-dimensional cell be a topological space that is homeomorphic to a closed $n$-ball. A CW complex may be constructed inductively from sets of these cells, where the $n$-skeleton of the CW complex is the union of the cells with dimension at most $n$. The process begins with the 0 -skeleton, or a set of points in the ambient space. The 1-skeleton is constructed from the set of 1-cells and the 0-skeleton by gluing the endpoints of the 1-cells to the 0 -skeleton. Generally speaking, the $n$-skeleton is constructed by attaching the set of $n$-cells to the $(n-1)$-skeleton by a quotient map from the boundary of the $n$-cells to the $(n-1)$-skeleton. The CW complex is regular if all of the attaching maps are homeomorphisms.

Our feeling, and we hope that of the referee as well, is that including this definition of the objects being studied would not serve the general reader any better than the more intuitive explanations already provided in the first paragraph of section 2 and the second paragraph of section 3. On the other hand, the definition of these topological objects is actually quite precise. Our intention in the final paragraph of the introduction was to strike a balance between the general reader and the reader desiring more mathematical rigor and precision, as is apparently the case with the referee. 
Upon further reflection, the reader who does not know the definition of a regular CW complex but who nevertheless is knowledgeable of mathematics may find the phrase "mathematically inclined reader" to be offensive. For this reason, we have changed the phrase to "readers with an understanding of topology" in an effort to avoid this situation.

The central concern that the referee expresses in this comment may not apply to the paper though. Defining a class of cell complexes with an associated weight function would absolutely be the way to proceed if the intention was to give a closed form expression for the expectation value of $g_{n}$. There is some precedent for this in the literature, as the referee will observe in the third paragraph of the introduction. Our purpose, on the other hand, is to derive an expression for $g_{n}$ that is not closed form but applies to any cell complex satisfying the topological requirements of a regular CW complex. That this is our intention is openly described in the fifth paragraph of the introduction. One advantage of this more general approach is the ability to identify quantities, like the average excess curvature, that may be used to construct specific classes of cell complexes of the type the referee describes. For this reason, we regard this comment of the referee as an insightful suggestion for the direction of future research in this area, and regret that the investigation of this issue is outside the confines of our paper.

\section{REFERENCES}

[1] Emanuel A. Lazar, Robert D. MacPherson, and David J. Srolovitz. A more accurate two-dimensional grain growth algorithm. Acta Mater., 58(2):364-372, January 2010.

[2] Emanuel A. Lazar, Jeremy K. Mason, Robert D. MacPherson, and David J. Srolovitz. A more accurate three-dimensional grain growth algorithm. Acta Materialia, 59(17):6837 - 6847, 2011.

[3] J. von Neumann. Written discussion. In Metal Interfaces, pages 108-110, Cleveland, Ohio, 1952. American Society for Metals.

[4] W.W. Mullins. Two-dimensional motion of idealized grain boundaries. Journal of Applied Physics, 27:900-904, March 1956.

[5] R.D. MacPherson and D.J. Srolovitz. The von Neumann relation generalized to coarsening of threedimensional microstructures. Nature, 446(7139):1053-1055, April 2007.

[6] D Weaire. Some remarks on the arrangement of grains in a polycrystal. Metallography, 7(2):157-160, 1974.

[7] D.A. Aboav. The arrangement of cells in a net. Metallography, 13(1):43-58, 1980. 


\title{
A geometric formulation of the Law of Aboav-Weaire in two and three dimensions
}

\author{
J. K. Mason ${ }^{1,2}$, R. Ehrenborg ${ }^{3}$ and E. A. Lazar ${ }^{4}$ \\ 1 School of Mathematics, Institute for Advanced Study, Princeton, NJ 08540, USA. \\ 2 Lawrence Livermore National Lab, Livermore, CA 94550, USA. \\ 3 Department of Mathematics, University of Kentucky, Lexington, KY 40506, USA. \\ 4 Program in Applied and Computational Mathematics, Princeton University, \\ Princeton, NJ 08544, USA. \\ E-mail: jkylemason@gmail.com
}

\begin{abstract}
The Law of Aboav-Weaire relates the number of sides of a grain to the average number of sides of the neighboring grains and is usually given as an empirical observation and restricted to two-dimensional systems. Certain attempts have been made to make this statement rigorous, or to extend the observation to three dimensions. This paper places the rule on a more solid foundation in two and three dimensions using geometric arguments. Specifically, we derive exact local forms of the Law of Aboav-Weaire, and an exact global form that is identical in two and three dimensions except for a single parameter $\zeta$. The derivation requires the definition and investigation of the average excess curvature, a previously unconsidered physical quantity. An approximation to our exact result is compared to the results of extensive simulations in two and three dimensions, and we provide a compact expression that strikes a balance between complexity and accuracy.
\end{abstract}

PACS numbers: $61.50,02.40,02.70$

Submitted to: J. Phys. A: Math. Gen.

\section{Introduction}

Several decades ago, while studying sections of polycrystalline $\mathrm{MgO}$ Aboav observed that grains with a small number of sides tended to be surrounded by grains with a large number of sides, and vice versa [1]. While this observation was initially suggested as evidence for a spatial ordering of the grains, a few years afterward Weaire [2] reasoned on the basis of Euler's Theorem that the average number of sides of the neighbors of an $n$-sided grain should be roughly

$$
g_{n} \approx 5+\frac{6+\mu_{2}}{n}
$$

where $\mu_{2}$ is the variance of the distribution of the number of sides of a grain. Since this prediction is fundamentally empirical, many researchers have proposed slight 
modifications to the above relation $[3,4,5,6,7]$ as measurements have been repeated and improved. Nevertheless, the clarity of Weaire's argument and the remarkable robustness of this relationship for a variety of two-dimensional systems $[3,8,9,10,11]$ has elevated something similar to the above expression to the status of a law.

While the Law of Aboav-Weaire is simple to state, a theoretical justification for this relation has proven more elusive. Prior approaches have employed geometric or combinatorial methods $[2,12,13,14]$, microreversibility of topological transformations $[8,12,15]$, the statistical mechanics formalism $[6,16]$, or application of the Palm method [5]. While all of these contribute to our understanding of the source of the Law of Aboav-Weaire, none of them is completely satisfactory. The explanations based on geometric or combinatorial methods require either that the correlations in turning angles decay exceedingly quickly $[2,13]$ or that $\mu_{2}$ be vanishingly small $[12,14]$, though there seems to be no physical basis for these assumptions. The realization that edge flips may occur more frequently on the boundary of grains with many sides [16] refutes the justifications based on the microreversibility of topological transformations, and Chiu [17] calls into question the assertion that the maximum entropy method from statistical mechanics is sufficient to derive the above relation. Finally, we must mention that several exact relations that reduce to the Law of Aboav-Weaire do appear in the literature [5, 12]. However, these themselves depend on functions of an unknown form and without a natural geometric interpretation, and which in practice must either be neglected completely or measured empirically.

Part of the difficulty with justifying the Law of Aboav-Weaire is precisely the assertion that this relation holds, to a good approximation, for all natural cell complexes. Several authors have been able to derive exact expressions that are similar to the above relation by restricting themselves to specific systems or classes of systems. For instance, this is the case for two-dimensional systems where the only allowed topological transformation is an edge flip [18] and for two-dimensional laminated networks [19]. We should mention that there is an asymptotic expression for large $n$ that applies to a Voronoi tessellation of points sampled from a Poisson distribution [7] as well, though this only applies to $n$ outside the practically measurable range.

Since most physically occurring microstructures and cell complexes are threedimensional, there is significant interest in formulating a relation comparable to the Law of Aboav-Weaire for these systems as well. This is considerably more difficult in three dimensions though, due to the relative scarcity of empirical results and to the increased complexity of the cell complexes involved. Fortes obtained an expression for the expected number of faces of a grain neighboring a grain with $f$ faces [20], and while his result applies only to the staggered stacking of prisms, he suggests that something of the same form is the natural analogue to the Law of Aboav-Weaire. Other arguments for a relation of the form proposed by Fortes appear in the literature [14], and some evidence that this may hold more generally is offered by simulations of a variety of threedimensional systems $[21,22,23,24]$. We mention that the result of Chiu [5] implies the suggestion of Fortes, though as before this comes at the price of defining a subsidiary 
function of unknown form and obscure geometric meaning.

Our purpose in this paper is not to 'prove' the Law of Aboav-Weaire in two and three dimensions for arbitrary cell complexes. Indeed, this should be regarded as a fruitless endeavor, since the above relation is known to not hold for certain cell complexes, including the Voronoi tessellation of points sampled from a Poisson distribution $[4,25]$. Rather, our intention is to provide a geometric derivation of an exact relation that separates into a part that is required for any cell complex and a part that depends on the characteristics of the particular cell complex being considered. We identify this second component as the 'average excess curvature', defined in Section 4. Furthermore, expanding the average excess curvature as a function of $n$ is found to give several of the proposed forms of the Law of Aboav-Weaire found in the literature. Specifically, we find that

$g_{n}=\langle n\rangle-a+\frac{\langle n\rangle \cdot a+\mu_{2}}{n}-\frac{1}{n} \cdot \frac{1}{\zeta} \cdot \sum_{k=2}^{\infty} c_{k} \cdot\left[(n-\langle n\rangle)^{k}-\mu_{k}\right]$

where $\langle n\rangle$ is the average number of sides of a grain, $\mu_{k}$ is the $k$ th central moment of the distribution of the number of sides, $\zeta$ is an angle that depends on the dimension, and $a$ and $c_{k}$ are constants that depend on the cell complex. We compare this expansion with the results from extensive simulations in two and three dimensions, and find an expression for the average excess curvature that strikes a balance between complexity and accuracy. In this process numerous other results are obtained as well, including several exact local forms of the Law of Aboav-Weaire and a unified expression that holds in two and three dimensions.

Finally, readers with an understanding of topology should be informed that throughout this paper, the phrase 'cell complex' refers to a regular CW complex.

\section{Two dimensions}

Consider a two-dimensional cell complex $\Omega$ covering the plane $\mathbb{R}^{2}$. An individual grain is the basic two-dimensional constituent of this structure, and may correspond to a grain in a microstructure, a bubble in a foam, or a cell in a biological tissue. Adjacent grains meet on edges, and adjacent edges meet at vertices. The cell complex $\Omega$ is called simple when exactly three edges and three grains meet at every vertex. An example of a simple cell complex is given in figure 1 .

Within the cell complex $\Omega$, consider $R$ to be an arbitrary finite collection of contiguous grains. Let $e_{j}^{i}$ be the $i$ th $j$-dimensional cell of $R$, where a grain is a 2dimensional cell, an edge is a 1-dimensional cell, and a vertex is a 0 -dimensional cell. Let $f_{i}(R)$ be the number of $i$-dimensional cells of $R$, and $f_{i, j}(R)$ be the number of pairs of an $i$-dimensional cell and a $j$-dimensional cell where the $i$-dimensional cell is contained in the boundary of the $j$-dimensional cell. These quantities should all be regarded as components of the more general flag $f$-vector [26].

For the remainder of this section, we place certain constraints on the geometry of

the cell complex $\Omega$. Rather than assuming that the cell complex be composed of straight 
edges, we allow the edges to be curved but require that edges meet at vertices at angles of $2 \pi / 3$. This is a natural constraint for cell complexes where the energy per unit area is directly proportional to the length of the edges in that area, as is the case for grain boundaries in a two-dimensional microstructure with uniform boundary energies. This constraint does not significantly limit the types of cell complexes to which our analysis applies, since deformations in small neighborhoods around the vertices may be used to bring a cell complex with arbitrary turning angles into this condition.

Lemma 1 (A Combinatorial Curvature). Let $\Omega$ be a simple cell complex subdividing the two-dimensional plane $\mathbb{R}^{2}$, where $\Omega$ is subject to the angular condition described above. Let $R$ be a finite collection of grains of $\Omega$, and let $\partial R$ be the boundary of $R$. Then the relation

$$
\int_{\partial R} \kappa \mathrm{d} s=2 \pi \cdot f_{2}(R)-\frac{\pi}{3} \cdot f_{0,2}(R)
$$

holds, where the quantity on the left is the integral of the curvature along the edges of $\partial R$.

Notice that the quantity on the right of (1) is directly proportional to the difference of six and the average number of vertices per grain in the region $R$. In particular, if $R$ is a single grain $G$, the integral of the curvature over the edges of $G$ vanishes when $G$ has six vertices. On the other hand, consider a region $R$ containing $N$ grains. Intuitively, as $N$ increases, the integral on the left should become small relative to $N$, suggesting that the average number of vertices of a grain is six.

Proof of Lemma 1: Let $D$ be a region in $\mathbb{R}^{2}$ with boundary $\partial D$ that is a piecewise smooth curve. The Gauss-Bonnet Theorem states that

$$
\int_{\partial D} \kappa \mathrm{d} s+\sum_{i} \alpha_{i}=2 \pi \cdot \chi(D)
$$

where the signed curvature $\kappa$ is integrated over the edges of $\partial D, \alpha_{i}$ is the exterior angle where the edges of $\partial D$ meet at the $i$ th vertex, and $\chi(D)$ is the Euler characteristic of $D$. For a single grain $G$ of $\Omega$, this becomes

$$
\int_{\partial G} \kappa \mathrm{d} s=2 \pi-\frac{\pi}{3} \cdot f_{0}(G)
$$

where $f_{0}(G)$ is the number of vertices of the grain $G$, the exterior angle for every vertex is exactly $\pi / 3$, and $\chi(G)$ is unity. Applying this relation to all of the grains of the region $R$ and summing gives (1). The integral of the curvature is performed only over the boundary $\partial R$ of the region $R$, since the integrals of the curvature along both sides of an internal edge cancel.

Theorem 2 (The Local Law of Aboav-Weaire). Let $G$ be a grain with $n$ neighboring grains and $R$ be the region composed of the grain $G$ and all of its neighbors. Let $m_{i}$ be 
the number of edges of the ith neighbor. Assuming that $m_{i} \geq 3$ for all $i$, the average number of edges per grain of the neighboring grains of $G$ is given by

$$
\frac{1}{n} \cdot \sum_{i=1}^{n} m_{i}=5+\frac{6}{n}-\frac{1}{n} \cdot \frac{3}{\pi} \cdot \int_{\bigcup_{\partial R} e_{1}} \kappa \mathrm{d} s .
$$

As a rough approximation, one may assume that the magnitude of the integral is generally small to find that

$$
\frac{1}{n} \cdot \sum_{i=1}^{n} m_{i} \approx 5+\frac{6}{n}
$$

Proof of Theorem 2: Since each grain has the same number of vertices as edges, we have the relation

$$
f_{1,2}(R)=f_{0,2}(R)
$$

Furthermore, provided that $m_{i} \geq 3$ for all $i$, or equivalently that $G$ does not border any two-edged grains, we have the relations

$$
\begin{aligned}
& f_{2}(R)=n+1 \\
& f_{1,2}(R)=n+\sum_{i=1}^{n} m_{i} .
\end{aligned}
$$

With the intention of eventually separating a quantity equal to the right side of (1), we add (6) to (7) multiplied by 6 to find that

$$
f_{1,2}(R)=6 n+6-\frac{3}{\pi} \cdot\left[2 \pi \cdot f_{2}(R)-\frac{\pi}{3} \cdot f_{0,2}(R)\right] .
$$

Substituting for $f_{1,2}(R)$ using $(8)$ and rearranging the result gives

$$
\frac{1}{n} \cdot \sum_{i=1}^{n} m_{i}=5+\frac{6}{n}-\frac{1}{n} \cdot \frac{3}{\pi} \cdot\left[2 \pi \cdot f_{2}(R)-\frac{\pi}{3} \cdot f_{0,2}(R)\right]
$$

which is the combinatorial form of the local Law of Aboav-Weaire. Finally, applying Lemma 1 gives the desired equality.

One advantage of this result is that (4) is exact for any given region $R$ that satisfies the assumptions of Theorem 2 and is part of a simple cell complex with the specified vertex angles. For instance, (4) remains valid when the region $R$ contains holes due to the arrangement of the grains, as in figure 1 . This situation is normally neglected in derivations of the Law of Aboav-Weaire. 


\section{Three dimensions}

Most natural cell complexes are three-dimensional. The literature generally assumes that there is an analogue of the Law of Aboav-Weaire for a three-dimensional cell complex, and furthermore that this analogue should provide a prediction for the average number of faces of the neighbors of an $n$-faced grain $[21,22,23,24]$. Historically though, empirical support for this assumption may be found only within simulation results, due to the practical difficulties of microscopy techniques able to resolve a three-dimensional microstructure. Moreover, the scarcity of direct experimental evidence and the increased complexity of the cell complex compared with the two-dimensional analogue has generally discouraged theoretical treatment of the three-dimensional version of the Law of Aboav-Weaire.

Consider a three-dimensional cell complex $\Omega$ covering three-dimensional space $\mathbb{R}^{3}$. In this section, a grain is the basic three-dimensional constituent of the cell complex. Adjacent grains meet on faces, adjacent faces meet on edges, and adjacent edges meet at vertices. The cell complex $\Omega$ is called simple when exactly three faces and three grains meet at every edge, and four edges, six faces and four grains meet at every vertex. An example of a simple cell complex is given in figure $2(\mathrm{~b})$.

Within the cell complex $\Omega$, consider $R$ to be an arbitrary finite collection of contiguous grains. Let $e_{j}^{i}$ be the $i$ th $j$-dimensional cell of $R$, where a grain is a 3 dimensional cell, a face is a 2-dimensional cell, an edge is a 1-dimensional cell, and a vertex is a 0 -dimensional cell. Let $f_{i}(R)$ and $f_{i, j}(R)$ be defined as for two dimensions. Finally, let $f_{i, j, k}(R)$ be the number of triplets of an $i$-dimensional cell, a $j$-dimensional cell and a $k$-dimensional cell where the $i$-dimensional cell is contained in the boundary of the $j$-dimensional cell, and the $j$-dimensional cell is contained in the boundary of the $k$-dimensional cell.

We place certain constraints on the geometry of the cell complex $\Omega$ for the remainder of this section. Specifically, we require that faces meet at edges at dihedral angles of $2 \pi / 3$ and that edges meet at vertices at angles of $\arccos (-1 / 3)$. This is a natural constraint for cell complexes where the energy per unit volume is directly proportional to the area of the faces contained in that volume, as is the case for a grain boundary network in a three-dimensional microstructure with uniform boundary energies. As before, deformations in small neighborhoods around the edges and vertices may be used to bring an arbitrary cell complex into this condition.

Lemma 3. Let $\Omega$ be a simple cell complex subdividing three-dimensional space $\mathbb{R}^{3}$ and let $R$ be a finite collection of grains of $\Omega$. Then the relation

$$
4 \cdot f_{3}(R)=2 \cdot f_{2,3}(R)-f_{0,3}(R)
$$

holds.

Proof of Lemma 3: The Euler relation for the boundary $\partial G$ of a single grain $G$ is

$$
2=f_{0}(G)-f_{1}(G)+f_{2}(G)
$$


where the Euler characteristic is 2 since the boundary of every grain is a spherical polyhedron. The property that $\Omega$ is simple imposes the condition $3 \cdot f_{0}(G)=f_{0,1}(G)=$ $2 \cdot f_{1}(G)$, causing the above expression to take the form

$$
4=2 \cdot f_{2}(G)-f_{0}(G) .
$$

Applying this relation to all of the grains of the region $R$ and summing gives (11).

Since we intend to roughly follow the same derivation as in Section 2, we require the equivalent of Lemma 1 for a three-dimensional cell complex. Let $D$ be a polyhedron $\ddagger$ in $\mathbb{R}^{3}$ with a piecewise smooth boundary $\partial D$. The supplementary material shows that the form of the Gauss-Bonnet Theorem suitable for this polyhedron is

$\int_{\partial D} \mathcal{K} e_{2} \mathrm{~d} A+\int_{\bigcup_{\partial D} e_{1}}(2-2 \cos \beta)^{1 / 2} \cdot \kappa \cdot \boldsymbol{e}_{2} \cdot \boldsymbol{v} \mathrm{d} s+\sum_{i} \alpha_{i}=2 \pi \cdot \chi(\partial D)$

where $\mathcal{K}$ is the Gaussian curvature of the faces, $\kappa$ is the curvature and $\boldsymbol{e}_{2}$ is the second Frenet vector of the edges, $\alpha_{i}$ is the angular defect of the $i$ th vertex, and $\chi(\partial D)$ is the Euler characteristic of $\partial D$. For the definition of the remaining quantities, consider the plane perpendicular to the tangent vector at a point along one of the edges of $\partial D$. Let $\beta$ be the turning angle of the curves of intersection of this plane with the neighboring faces, and let $\boldsymbol{v}$ be the unit vector in this plane that bisects the dihedral angle, as depicted in figure 3 .

Finally, we define the numerical constants $a_{0}=\pi-\arccos (-1 / 3)$ and $a_{1}=$ $2 \pi-3 \arccos (-1 / 3)$, which are the turning angle at a vertex of a face and the concentrated Gaussian curvature at a vertex of an isolated grain, respectively. With all of these definitions in hand, we supply the following lemma.

Lemma 4 (A Combinatorial Curvature). Let $\Omega$ be a simple cell complex subdividing $\mathbb{R}^{3}$, where the faces meet at dihedral angles of $2 \pi / 3$ and the edges meet at angles of $\arccos (-1 / 3)$. Let $R$ be a finite collection of grains $G_{i}$ of $\Omega$ indexed by $i$. Then the three relations

$\sum_{i=1}^{f_{3}(R)}\left[\int_{\bigcup_{\partial G_{i}} e_{2}} \mathcal{K} \mathrm{d} A+\int_{\bigcup_{\partial G_{i}} e_{1}} \kappa \cdot \boldsymbol{e}_{2} \cdot \boldsymbol{v} \mathrm{d} s\right]=\left\{\begin{array}{l}4 \pi \cdot f_{3}(R)-a_{1} \cdot f_{0,3}(R) \\ 2 \pi \cdot f_{2,3}(R)-a_{0} \cdot f_{0,2,3}(R) \\ 12 a_{0} \cdot f_{3}(R)-2 a_{1} \cdot f_{2,3}(R)\end{array}\right.$

hold, where the summand is given by the sum of the integral of the Gaussian curvature over the faces of the ith grain and the integral of the concentrated Gaussian curvature over the edges of the ith grain, and the summation is performed over all grains in the region $R$.

Notice that the first line on the right is directly proportional to the difference of 22.795 and the average number of vertices per grain in $R$, the second line on the right is directly proportional to the difference of 5.104 and the average number of vertices per ¥ A regular CW complex that is homeomorphic to the standard 3-ball. The boundary of the polyhedron is the inverse image of the standard 2-sphere. 
face in $R$, and the third line on the right is directly proportional to the difference of 13.397 and the average number of faces per grain in $R$. In particular, if $R$ is a single grain $G$, the integral of the Gaussian curvature over the faces and edges of $G$ vanishes when $G$ has 22.795 vertices and 13.397 faces. Since the number of vertices and faces of $G$ must be integers, the integral of the Gaussian curvature over the faces and edges of $G$ cannot vanish.

Proof of Lemma 4: For a single grain $G_{i}$ of $R$, the Gauss-Bonnet Theorem as expressed in (14) becomes

$$
\int_{\partial G_{i}} \mathcal{K} \mathrm{d} A+\int_{\bigcup_{\partial G_{i}} e_{1}} \kappa \cdot \boldsymbol{e}_{2} \cdot \boldsymbol{v} \mathrm{d} s=4 \pi-a_{1} \cdot f_{0}\left(G_{i}\right)
$$

where $f_{0}\left(G_{i}\right)$ is the number of vertices of the grain $G_{i}$ and $\chi\left(\partial G_{i}\right)$ is 2 because the boundary of every grain is a spherical polyhedron. The angular constraints placed on the cell complex $\Omega$ require that the turning angle $\beta$ always be $\pi / 3$, and that angular defect for every vertex always be $a_{1}$. Applying this relation to all of the grains of the region $R$ and summing gives the first equality of (15).

We prove the remaining two equalities of (15) by showing the equivalence of the combinatorial quantities on the right sides. The equivalence of the first and second lines on the right of (15) is found by using Lemma 3 to substitute for $f_{3}(R)$ in the first line, giving the relation

$$
4 \pi \cdot f_{3}(R)-a_{1} \cdot f_{0,3}(R)=2 \pi \cdot f_{2,3}(R)-\left(\pi+a_{1}\right) \cdot f_{0,3}(R) .
$$

Since $\pi+a_{1}=3 a_{0}$ and $3 \cdot f_{0,3}(R)=f_{0,2,3}(R)$, the second equality of $(15)$ holds. The equivalence of the first and third lines on the right of (15) is found by using Lemma 3 to substitute for $f_{0,3}(R)$ in the first line, giving the relation

$$
4 \pi \cdot f_{3}(R)-a_{1} \cdot f_{0,3}(R)=\left(4 \pi+4 a_{1}\right) \cdot f_{3}(R)-2 a_{1} \cdot f_{2,3}(R) .
$$

Since $4 \pi+4 a_{1}=12 a_{0}$, the third equality of (15) holds.

Theorem 5. Let $G$ be a grain with $n$ neighboring grains and $R$ be the region composed of the grain $G$ and all of its neighbors. Let $m_{i}$ be the number of faces of the ith neighbor. Assuming that no two-edged faces share exactly one edge with $G$, the average number of faces per grain of the neighboring grains of $G$ is given by

$$
\begin{aligned}
\frac{1}{n} \cdot \sum_{i=1}^{n} m_{i}= & \left(\frac{2 \pi}{a_{1}}+1\right)+\frac{1}{n} \cdot\left(\frac{2 \pi}{a_{1}}+2\right) \\
& -\frac{1}{n} \cdot \frac{1}{2 a_{1}} \cdot \sum_{j=1}^{n+1}\left[\int_{\bigcup_{\partial G_{j}} e_{2}} \mathcal{K} \mathrm{d} A+\int_{\bigcup_{\partial G_{j}} e_{1}} \kappa \cdot \boldsymbol{e}_{2} \cdot \boldsymbol{v} \mathrm{d} s\right]
\end{aligned}
$$

where $G_{j}$ is the jth grain of $R$. 
As a rough numerical approximation, one may assume that the magnitude of the integral is generally small to find that

$$
\frac{1}{n} \cdot \sum_{i=1}^{n} m_{i} \approx 12.397+\frac{13.397}{n}
$$

Proof of Theorem 5: Since, by assumption, no two-edged faces share exactly one edge with $G$, we have the relations

$$
\begin{aligned}
& f_{3}(R)=n+1, \\
& f_{2,3}(R)=n+\sum_{i=1}^{n} m_{i} .
\end{aligned}
$$

With the intention of eventually separating a quantity equal to the right side of (15), we divide (11) by two and rearrange the result to find that

$$
f_{2,3}(R)=\left(\frac{2 \pi}{a_{1}}+2\right) \cdot f_{3}(R)-\frac{1}{2 a_{1}}\left[4 \pi \cdot f_{3}(R)-a_{1} \cdot f_{0,3}(R)\right] .
$$

Substituting for $f_{3}(R)$ and $f_{2,3}(R)$ using (21) and (22) respectively, solving for the summation and dividing through by $n$ gives

$\frac{1}{n} \cdot \sum_{i=1}^{n} m_{i}=\left(\frac{2 \pi}{a_{1}}+1\right)+\frac{1}{n} \cdot\left(\frac{2 \pi}{a_{1}}+2\right)-\frac{1}{n} \cdot \frac{1}{2 a_{1}} \cdot\left[4 \pi \cdot f_{3}(R)-a_{1} \cdot f_{0,3}(R)\right]$

which is one of the combinatorial forms of the analogue to Theorem 2. The other combinatorial forms may be found by substituting one of the other quantities on the right side of (15) for the quantity in brackets. Finally, applying Lemma 4 gives the desired equality.

Notice that (19) is exact for any particular region $R$ that satisfies the assumptions of Theorem 5 and is part of a simple cell complex with the specified dihedral angles and vertex angles. In particular, as with the two-dimensional case, (19) remains valid when the region $R$ contains holes due to the arrangement of the grains.

\section{The Global Relations}

While the results of Sections 2 and 3 apply to the average number of faces per grain of the neighbors around a particular grain, the usual form of the Law of Aboav-Weaire applies to the average number of faces per grain of the neighbors around an average grain with a specified number of neighbors [2, 3]. We refer to these predictions as the global relations to distinguish them from the local relations of earlier sections.

Consider a simple two-dimensional cell complex $\Omega$ as in Section 2. Let $N_{n}$ be the number of grains in $\Omega$ with $n$ neighbors, and let $H_{i}$ be the $i$ th grain with $n$ neighbors. 
Let $S_{i}$ be the region consisting of $H_{i}$ and all neighboring grains, and let $m_{i, j}$ be the number of edges of the $j$ th neighbor of $H_{i}$. The average of (10) over all $H_{i}$ is

$$
\frac{1}{N_{n}} \cdot \sum_{i=1}^{N_{n}}\left[\frac{1}{n} \cdot \sum_{j=1}^{n} m_{i, j}\right]=5+\frac{6}{n}-\frac{1}{n} \cdot \frac{3}{\pi} \cdot \frac{1}{N_{n}} \cdot \sum_{i=1}^{N_{n}}\left[2 \pi \cdot f_{2}\left(S_{i}\right)-\frac{\pi}{3} \cdot f_{0,2}\left(S_{i}\right)\right] .
$$

Let $p_{n}$ be the frequency of grains with $n$ neighbors in $\Omega$, and let $\langle\cdot\rangle=\sum_{n}\left(p_{n} \cdot\right)$ indicate the average of a quantity over $n$. We write the average of the quantity in brackets on the right as

$\frac{1}{N_{n}} \cdot \sum_{i=1}^{N_{n}}\left[2 \pi \cdot f_{2}\left(S_{i}\right)-\frac{\pi}{3} \cdot f_{0,2}\left(S_{i}\right)\right]=2 \pi \cdot(n+1)-\frac{\pi}{3} \cdot\langle n\rangle \cdot(n+1)+\epsilon_{n}$

where $\epsilon_{n}$ is referred to as the average excess curvature, and is defined by the above equation. Notice that by Lemma 1, the quantity on the left is the expectation value of the sum of the integrals of the curvature over the boundaries of the $n+1$ grains in any given $S_{i}$. Meanwhile, by the same lemma, the expression $2 \pi \cdot(n+1)-\pi / 3 \cdot\langle n\rangle \cdot(n+1)$ is the expectation value of the sum of the integrals of the curvature over the boundaries of $n+1$ grains, chosen independently and randomly from the entire population in $\Omega$. This means that $\epsilon_{n}$ is the difference of the expectation values of the integrated curvature over the boundaries of two populations of grains, one given by a cluster around a central grain with $n$ neighbors, and the other by the same number of grains chosen randomly from the cell complex. Substituting (26) into (25) and multiplying through gives

$$
g_{n}=5+\frac{6}{n}-\left(6+\frac{6}{n}-\langle n\rangle-\frac{\langle n\rangle}{n}+\frac{1}{n} \cdot \frac{3}{\pi} \cdot \epsilon_{n}\right)
$$

where $g_{n}$ is the average number of edges per grain around an average grain with $n$ neighbors. The average excess curvature $\epsilon_{n}$ is defined to allow us to cancel terms deriving from the local constrains and replace them with the corresponding global quantities, leaving

$$
g_{n}=\langle n\rangle-1+\frac{\langle n\rangle}{n}-\frac{1}{n} \cdot \frac{3}{\pi} \cdot \epsilon_{n}
$$

for the Law of Aboav-Weaire. Since $\langle n\rangle$ is precisely 6 in two dimensions, this exchange amounts to a convenient change in notation. Notice that the average excess curvature not only completely specifies $g_{n}$, but is the single quantity that depends on the specific features of the cell complex $\Omega$.

The procedure followed above is nearly the same for the simple three-dimensional cell complex of Section 3. Begin with (19), where the third line of (15) is substituted for the quantity in brackets on the right. Let $N_{n}, H_{i}$ and $S_{i}$ be defined as for the two-dimensional case, and let $m_{i, j}$ be the number of faces of the $j$ th neighbor of $H_{i}$. Averaging over all $H_{i}$ gives

$$
\begin{aligned}
\frac{1}{N_{n}} \cdot \sum_{i=1}^{N_{n}}\left[\frac{1}{n} \cdot \sum_{j=1}^{n} m_{i, j}\right]= & \left(\frac{2 \pi}{a_{1}}+1\right)+\frac{1}{n} \cdot\left(\frac{2 \pi}{a_{1}}+2\right) \\
& -\frac{1}{n} \cdot \frac{1}{2 a_{1}} \cdot \frac{1}{N_{n}} \cdot \sum_{i=1}^{N_{n}}\left[12 a_{0} \cdot f_{3}\left(S_{i}\right)-2 a_{1} \cdot f_{2,3}\left(S_{i}\right)\right] .
\end{aligned}
$$


The quantity in brackets on the right is equal to the sum of the integrals of the Gaussian curvature over the faces of the $n+1$ grains in $S_{i}$. We write the average as

$$
\frac{1}{N_{n}} \cdot \sum_{i=1}^{N_{n}}\left[12 a_{0} \cdot f_{3}\left(S_{i}\right)-2 a_{1} \cdot f_{2,3}\left(S_{i}\right)\right]=12 a_{0} \cdot(n+1)-2 a_{1} \cdot\langle n\rangle \cdot(n+1)+\epsilon_{n}
$$

where we abuse notation by using the same symbols to indicate analogous quantities in two and three dimensions. The average excess curvature $\epsilon_{n}$ in three dimensions is the difference of the expectation values of the integrated Gaussian curvature over the boundaries of two populations of grains, one given by a cluster around a central grain with $n$ neighbors, and the other by the same number of grains chosen independently and randomly from all of $\Omega$. Substituting (30) into (29) allows us to cancel the quantities expressing local conditions and replace them with the corresponding global ones, resulting in

$$
g_{n}=\langle n\rangle-1+\frac{\langle n\rangle}{n}-\frac{1}{n} \cdot \frac{1}{2 a_{1}} \cdot \epsilon_{n}
$$

for grains in the three-dimensional cell complex. Notice that $\langle n\rangle$ is not generally 13.397 in three dimensions. Indeed, $\langle n\rangle$ may vary considerably from one cell complex to another, though our simulations [27] suggest a value of roughly $13.769 \pm 0.016$ for the steady-state condition.

The striking similarity of (28) and (31) strongly encourages that these results be unified in a single equation

$$
g_{n}=\langle n\rangle-1+\frac{\langle n\rangle}{n}-\frac{1}{n} \cdot \frac{1}{\zeta} \cdot \epsilon_{n}
$$

where $\zeta$ is an angle depending on the dimension. Since this equation is still exact, the derivation of the Law of Aboav-Weaire for a cell complex in two or three dimensions is reduced to the derivation or measurement of the average excess curvature $\epsilon_{n}$. We should point out that (32) is quite similar to the exact result of Chiu [5], though Chiu did not give a physical or geometric interpretation to the quantity in his result that is analogous to our average excess curvature. Indeed, this should be expected, because while his derivation extends to dimensions higher than three, our derivation is considerably more geometric.

One constraint on $\epsilon_{n}$ may be derived by a counting argument. For a given grain and an integer $n$, assign to the grain an integer weight that indicates the number of neighboring grains with $n$ faces. The quantity $N_{n} \cdot n \cdot g_{n}$ is the sum of the weighted numbers of faces of all the grains in the cell complex. Consider the sum of $N_{n} \cdot n \cdot g_{n}$ over all $n$, and let $G$ be an arbitrary grain with $m$ neighboring grains. The $m$ faces of $G$ must be included in this sum with a multiplicity of $m$, once for each neighbor of $G$. This implies the equality

$$
\sum_{n} N_{n} \cdot n \cdot g_{n}=\sum_{n} N_{n} \cdot n^{2} .
$$


Using the proportionality of $N_{n}$ and $p_{n}$ and the notation for averages introduced earlier in this section, one finds that

$$
\left\langle n \cdot g_{n}\right\rangle=\left\langle n^{2}\right\rangle
$$

which is equivalent to an earlier observation of Weaire [2]. Finally, using (32) to substitute for $g_{n}$ and simplifying the result gives

$$
-\frac{1}{\zeta}\left\langle\epsilon_{n}\right\rangle=\mu_{2}
$$

as an equation constraining the average excess curvature, where $\mu_{2}$ is the variance of the distribution of the number of faces of a grain.

Since the functional form of the average excess curvature is not known, we simply assume that $\epsilon_{n}$ may be expanded as a Taylor series in $n-\langle n\rangle$. That is, we assume that

$$
\epsilon_{n}=\sum_{k=0}^{\infty} c_{k} \cdot(n-\langle n\rangle)^{k}
$$

for some coefficients $c_{k}$ and for all observable $n$. We satisfy the prior constraint on the average excess curvature by substituting (36) into (35) and exchanging the order of the summations. On solving for the coefficient $c_{0}$ and substituting the result into (36), the general expression for the average excess curvature becomes

$$
\epsilon_{n}=-\zeta \cdot \mu_{2}+\sum_{k=1}^{\infty} c_{k} \cdot\left[(n-\langle n\rangle)^{k}-\mu_{k}\right]
$$

where $\mu_{k}$ is the $k$ th central moment of the distribution of the number of sides. Despite certain questions of convergence, (37) is quite useful for comparisons with prior results in the literature. Substituting (37) into (32) reveals that

$g_{n}=\langle n\rangle-a+\frac{\langle n\rangle \cdot a+\mu_{2}}{n}-\frac{1}{n} \cdot \frac{1}{\zeta} \cdot \sum_{k=2}^{\infty} c_{k} \cdot\left[(n-\langle n\rangle)^{k}-\mu_{k}\right]$

where $a=1+c_{1} / \zeta$. At this point, setting $c_{k}=0$ for $k>0$ gives the form of the Law of Aboav-Weaire suggested by Weaire [2], while setting $c_{k}=0$ for $k>1$ gives the one proposed by Aboav [3]. These correspond to constant and linear approximations of the average excess curvature, respectively. Now, the Law of Aboav-Weaire is usually applied to two-dimensional cell complexes with $n$ ranging from 3 to 14 , and is usually written following Aboav [3]. We point out that a linear model is a good approximation of any smooth function over a small domain, and that the success of the Law of Aboav-Weaire does not necessarily indicate that the average excess curvature is actually linear in $n$.

There does appear a third form in the literature corresponding to (38). Boots [4] found that the Law of Aboav-Weaire could be modified to hold for a Voronoi tessellation of points sampled from a Poisson distribution by including a term proportional to $n^{-2}$. Le Caër [28] found that the same expression performed well for Voronoi tessellations of the eigenvalues of complex Gaussian random matrices, and Kumar [13] provided some justification for the additional term. Since $\epsilon_{n}$ is expanded as a Taylor series, this form 
of the Law of Aboav-Weaire may be considered to arise from our results as well for suitable values of the $c_{k}$.

Finally, we point out that Taylor's Theorem does not strictly apply to (38). That is, setting $c_{k}=0$ for $k>K$ will give an error that is of order a constant in $n$ for any $K$, even though we provide empirical evidence in the following section that the approximation improves as $K$ increases.

\section{Numerical Results}

There remains the question of the number of terms to retain from (37) and (38) for the resulting approximations to $\epsilon_{n}$ and $g_{n}$ to be accurate enough for practical use. We consider four cases in this section. The first is obtained by setting $c_{k}=0$ for $k>1$, corresponds to the form of the Law of Aboav-Weaire given by Aboav [3], and is plotted in red. The second is given by setting $c_{k}=0$ for $k>2$ and is plotted in blue, the third is found by setting $c_{k}=0$ for $k>3$ and is plotted in green, and the fourth is found by setting $c_{k}=0$ for $k>4$ and is plotted in cyan. These final three conditions do not seem to appear in the literature.

We evaluate the ability of these approximations to follow the trends in the measured values from four simulated cell complexes. These include two- and three-dimensional versions of a Voronoi tessellation of points sampled from a Poisson distribution, and two- and three-dimensional versions of a simulated microstructure evolved via normal grain-growth to the steady-state regime. Our simulations of normal grain-growth move a discretized grain boundary in accordance with local versions of the von NeumannMullins relation [29, 30] and the MacPherson-Srolovitz relation [31] in two and three dimensions, respectively. At the point that the statistical features of the microstructure no longer measurably change with continued grain coarsening, the cell complex is regarded as being in the steady-state. Further details of the implementation of this algorithm are provided by Lazar [32, 27]. The two-dimensional cell complexes contain slightly more than $10^{6}$ grains each, and the three-dimensional cell complexes contain slightly more than $10^{5}$ grains each. The mean values of $\epsilon_{n}$ and $g_{n}$ for a given $n$ appear as black dots, with error bars indicating $95 \%$ confidence intervals on the location of the mean according to the the Vysochanskij-Petunin inequality.

The usual practice in the literature would be to fit the various approximations for $\epsilon_{n}$ and $g_{n}$ to the mean values directly, though this does not account for the radical variation in the confidence intervals with the value of $n$. This paper follows a more statistically robust procedure, and considers a set of grains that neighbors a particular grain with $n$ faces as providing a single measurement of $\epsilon_{n}$ and $g_{n}$. We find the coefficients that minimize the sum of the squared distances from the given approximation to the $10^{6}$ measured values for the two-dimensional cell complexes and $10^{5}$ measured values for the three-dimensional cell complexes. This effectively increases the weight for values of $n$ with many grains and decreases the weight for values of $n$ with few grains.

For all cases considered, the root-mean-square error strictly decreases as the number 
of terms retained from (37) and (38) is increased. That is, the mathematical difficulties described in Section 4 do not appear to be cause for particular concern. Since the quality of fit of $g_{n}$ depends entirely on the quality of fit of $\epsilon_{n}$, we concentrate on the graphs on the left of figures 4, 5, 6 and 7. The approximation of $\epsilon_{n}$ with $c_{k}=0$ for $k>1$, appearing in red, is observed to not follow the behavior of the measured values of $\epsilon_{n}$ at the extremal values of $n$. This deviation is particularly pronounced and systematic for figures 4 and 6, corresponding to the Voronoi tessellations of points sampled from a Poisson distribution in two and three dimensions. Setting $c_{k}=0$ for $k>2$ in the approximation gives the curves appearing in blue. This substantially reduces the rootmean-square error in all cases, except for the three-dimensional steady state structure where the change is negligible. While the root-mean-square error is further reduced for the green curves with $c_{k}=0$ for $k>3$ terms and for the cyan curves with $c_{k}=0$ for $k>4$, this improvement is offset by the complexity of introducing additional fitting parameters. We suggest that setting $c_{k}=0$ for $k>2$ is the appropriate balance between accuracy and complexity when applying the Law of Aboav-Weaire to an arbitrary cell complex. With $b=c_{2} / \zeta$, this gives

$$
g_{n}=\langle n\rangle \cdot(1+2 b)-a+\frac{\langle n\rangle \cdot a-\langle n\rangle^{2} \cdot b+\mu_{2} \cdot(1+b)}{n}-n \cdot b
$$

as our suggestion for a practical expression for $g_{n}$, provided explicitly for reference.

We point out that the approximations to $\epsilon_{n}$ given by the blue and green curves deviate from the measured values for $n>9$ in figure 5 . The reason for this is a curious variation in the measured values of $\epsilon_{n}$ for $5<n<8$ in the two-dimensional steady state structure. The presence of this variation seems to be a property of the steady state structure that is independent of dimension, since a similar variation is observed in figure 7. Specifically, the average excess curvature seems to be increased for values of $n$ near six in two dimensions and for values of $n$ near ten in three dimensions, that is, around the average value of $n$. Given our confidence intervals, this behavior does not appear to the result of stochasticity. Nevertheless, the reason for this phenomenon is not known, and we are not aware of any mention of this elsewhere in the literature.

\section{Conclusions}

We have derived exact local forms of the Law of Aboav-Weaire in two dimensions, and have performed an analogous derivation in three dimensions. We have further demonstrated that the corresponding global forms should reduce to a single expression, identical in two and three dimensions except for the single angular quantity $\zeta$. Identifying this expression required the definition of a further physical quantity, the average excess curvature, as defined in Section 4. Furthermore, numerical results obtained from grain growth simulations suggest a correction to the empirically derived Law of Aboav-Weaire. This correction extends the usefulness of the Law of Aboav-

Weaire to three-dimensional structures and to other cell complexes generally considered 
to be exceptions to the rule, including Voronoi tessellations of uniformly distributed points in two and three dimensions.

One point worthy of further study is the elevation of the average excess curvature in the steady-state structures for values of $n$ near six in two dimensions and for values of $n$ near ten in three dimensions. As far as we are aware the cause of this phenomenon is not known, and we hope that the meaning of this systematic deviation from the prevailing behavior of the average excess curvature will become clear in the future.

Finally, one may consider relaxing the conditions on the cell complex $\Omega$. For instance, the energetics of certain physical systems allow for the stable formation of vertices with four incident edges in two dimensions, or triple lines with four incident faces in three dimensions. This leads to contemplation of a corresponding relation for subdivisions of $\mathbb{R}^{2}$ or $\mathbb{R}^{3}$ that are not simple. Further afield, there is good reason to investigate the existence of analogous relations for subdivisions of other manifolds entirely, perhaps with varying intrinsic curvature, and whether the concept of average excess curvature extends in a natural way. While no longer physically motivated, one may ask all of the same questions for cell complexes on manifolds of dimension higher than three. Although the original empirical observation is already several decades old, there remains ample opportunity for research in this area.

\section{Acknowledgments}

The authors would like to thank the Institute for Advanced Study where this research was carried out, and especially Robert D. MacPherson for bringing us together. The first author was partially supported by grant HR0011-08-1-0093, and partially supported under the auspices of the U.S. Department of Energy by Lawrence Livermore National Laboratory under Contract DE-AC52-07NA27344. The second author was partially supported by National Science Foundation grants DMS-0902063, DMS-0835373 and CCF-0832797. The third author was supported by grant HR0011-08-1-0093.

\section{References}

[1] D A Aboav. The arrangement of grains in a polycrystal. Metallography, 3(4):383-390, 1970.

[2] D Weaire. Some remarks on the arrangement of grains in a polycrystal. Metallography, 7(2):157160, 1974.

[3] D A Aboav. The arrangement of cells in a net. Metallography, 13(1):43-58, 1980.

[4] B N Boots and D J Murdoch. The spatial arrangement of random Voronoi polygons. Comput. Geosci., 9(3):351-365, 1983.

[5] S N Chiu. Mean-value formulae for the neighbourhood of the typical cell of a random tessellation. Adv. Appl. Probab., 26(3):565-576, 1994.

[6] G Schliecker and S Klapp. Why are the equilibrium properties of two-dimensional random cellular structures so similar? Europhys. Lett., 48(2):122-128, October 1999.

[7] H J Hilhorst. Planar Voronoi cells: the violation of Aboav's law explained. J. Phys. A-Math. Gen., 39(23):7227-7243, June 2006.

[8] M Blanc and A Mocellin. Grain coordination in plane sections of polycrystals. Acta Metall. Mater., 27(7):1231-1237, 1979. 
[9] D A Aboav. The arrangement of cells in a net. II. Metallography, 16(3):265-273, 1983.

[10] B N Boots. Comments on Aboav Rule for the arrangement of cells in a network. Metallography, 17(4):411-418, 1984.

[11] J C Earnshaw and D J Robinson. Topological correlations in colloidal aggregation. Phys. Rev. Lett., 72(23):3682-3685, Jun 1994.

[12] C J Lambert and D L Weaire. Theory of the arrangement of cells in a network. Metallography, 14(4):307-318, 1981.

[13] S Kumar, SK Kurtz, and D Weaire. Average number of sides for the neighbors in a Poisson-Voronoi Tesselation. Philos. Mag. B, 69(3):431-435, March 1994.

[14] S F Edwards and K D Pithia. A note on the Aboav-Weaire law. Physica A, 205(4):577-584, 1994.

[15] N Rivier. Statistical crystallography-structure of random cellular networks. Philos. Mag. B, 52(3):795-819, 1985.

[16] M A Peshkin, K J Strandburg, and N Rivier. Entropic predictions for cellular networks. Phys. Rev. Lett., 67(13):1803-1806, September 1991.

[17] S N Chiu. A comment of Rivier's maximum-entropy method of statistical crystallography. J. Phys. A-Math. Gen., 28(3):607-615, February 1995.

[18] C Godrèche, I Kostov, and I Yekutieli. Topological correlations in cellular structures and planar graph theory. Phys. Rev. Lett., 69(18):2674-2677, Nov 1992.

[19] M A Fortes. Applicability of the Lewis and Aboav-Weaire laws to 2D and 3D cellular structures based on Pisson partitions. J. Phys. A-Math. Gen., 28(4):1055-1068, February 1995.

[20] M A Fortes. Topological properties of cellular structures based on the staggered packing of prisms. J. Phys.-Paris, 50(7):725-731, April 1989.

[21] M A Fortes. Applicability of Aboav's rule to a three-dimensional Voronoi partition. Phil. Mag. Lett., 68(2):69-71, August 1993.

[22] L Oger, A Gervois, J P Troadec, and N Rivier. Voronoi tessellation of packings of spheres: Topological correlation and statistics. Philos. Mag. B, 74(2):177-197, August 1996.

[23] F Wakai, N Enomoto, and H Ogawa. Three-dimensional microstructural evolution in ideal grain growth - General statistics. Acta Mater., 48(6):1297-1311, April 2000.

[24] S Jurine, S Cox, and F Graner. Dry three-dimensional bubbles: growth-rate, scaling state and correlations. Colloid. Surface. A, 263(1-3):18-26, August 2005.

[25] B N Boots. The arrangement of cells in random networks. Metallography, 15(1):53-62, 1982.

[26] M M Bayer and L J Billera. Generalized Dehn-Sommerville relations for polytopes, spheres and Eulerian partially ordered sets. Invent. Math., 79(1):143-157, 1985.

[27] E A Lazar, J K Mason, R D MacPherson, and D J Srolovitz. A more accurate three-dimensional grain growth algorithm. Acta Materialia, 59(17):6837 - 6847, 2011.

[28] G Le Caër and J S Ho. The Voronoi tessellation generated from eigenvalues of complex random matrices. J. Phys. A-Math. Gen., 23(14):3279-3295, July 1990.

[29] J von Neumann. Written discussion. In Metal Interfaces, pages 108-110, Cleveland, Ohio, 1952. American Society for Metals.

[30] W W Mullins. Two-dimensional motion of idealized grain boundaries. Journal of Applied Physics, 27:900-904, March 1956.

[31] R D MacPherson and D J Srolovitz. The von Neumann relation generalized to coarsening of three-dimensional microstructures. Nature, 446(7139):1053-1055, April 2007.

[32] Emanuel A. Lazar, Robert D. MacPherson, and David J. Srolovitz. A more accurate twodimensional grain growth algorithm. Acta Mater., 58(2):364-372, January 2010.

\section{Figures}




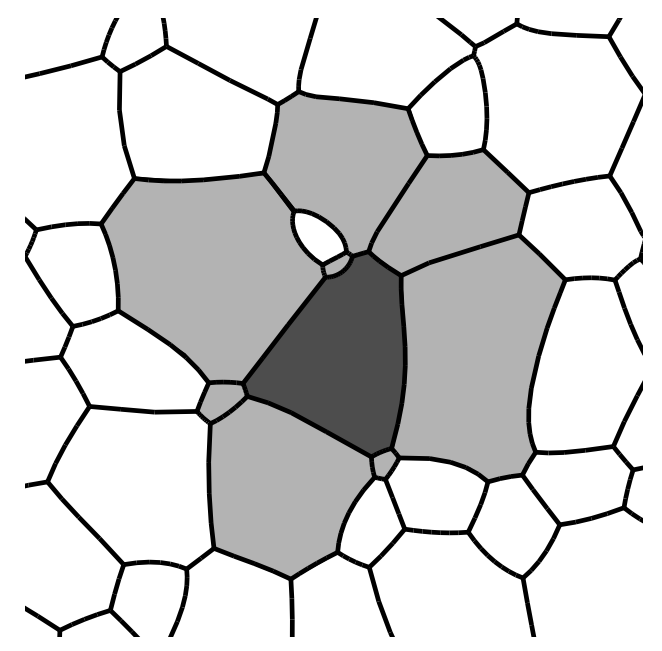

Figure 1. A small region of a simple cell complex covering the plane. Shaded in light grey is the region $R$ consisting of a grain $G$ in dark grey and all neighboring grains. While unusual, the region $R$ may contain any number of holes depending on the arrangement of the grains.
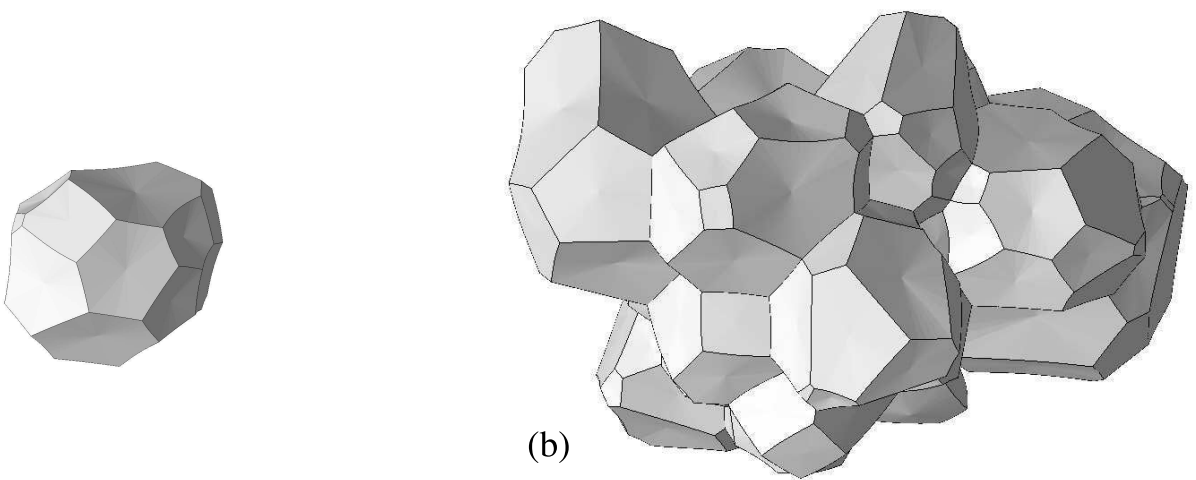

Figure 2. A depiction of (a) the grain $G$ and (b) the region $R$ as defined in Theorem 5 .

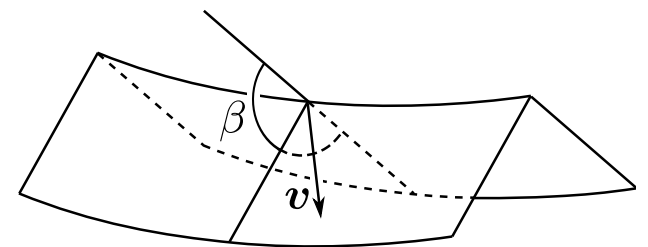

Figure 3. Definition of the unit vector $\boldsymbol{v}$ and turning angle $\beta$ necessary to find the Gaussian curvature concentrated at the curved edges of the boundary of a grain. 

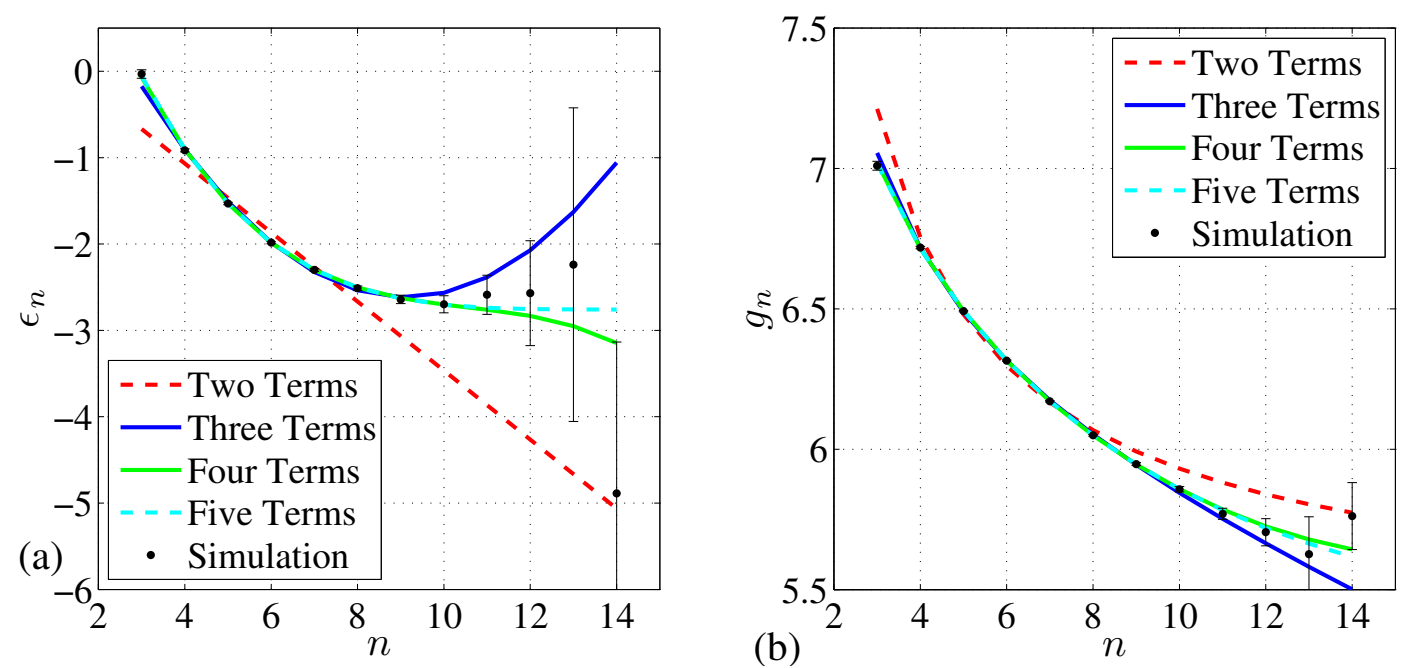

Figure 4. (a) $\epsilon_{n}$ and $g_{n}$ as functions of $n$ for the two-dimensional Poisson-Voronoi structure where $\mu_{2}$ is 1.7823 . Simulation results appear as black dots, while red, blue, green and cyan curves use values of $\epsilon_{n}$ given by least-squares fits of (37) with $c_{k}=0$ for $k$ greater than one, two, three and four, respectively. Fitting coefficients, given to the precision of the fitting procedure and ordered by increasing $k$, are -0.399542 for the red curves, -0.406900 and 0.0653253 for the blue curves, $-0.379467,0.071121$ and -0.0052241 for the green curves, and $-0.377280,0.069514,-0.0057421$ and 0.00017964 for the cyan curves. Error bars indicate $95 \%$ confidence intervals on the mean.
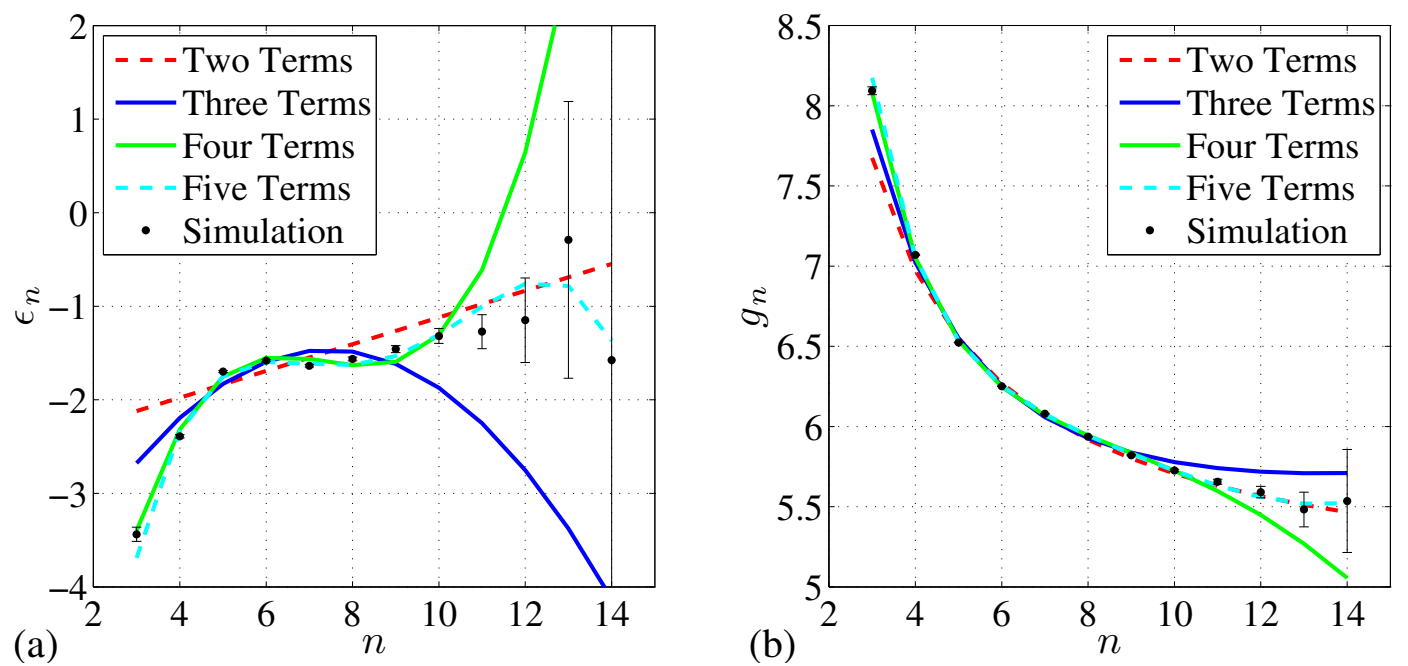

Figure 5. (a) $\epsilon_{n}$ and (b) $g_{n}$ as functions of $n$ for the two-dimensional steady state structure where $\mu_{2}$ is 1.6156 . Simulation results appear as black dots, while red, blue, green and cyan curves use values of $\epsilon_{n}$ given by least-squares fits of (37) with $c_{k}=0$ for $k$ greater than one, two, three and four, respectively. Fitting coefficients, given to the precision of the fitting procedure and ordered by increasing $k$, are 0.142942 for the red curves, 0.176895 and -0.0616744 for the blue curves, $0.068871,-0.104854$ and 0.0257262 for the green curves, and $0.031047,-0.083475,0.0365093$ and -0.00326445 for the cyan curves. Error bars indicate $95 \%$ confidence intervals on the mean. 

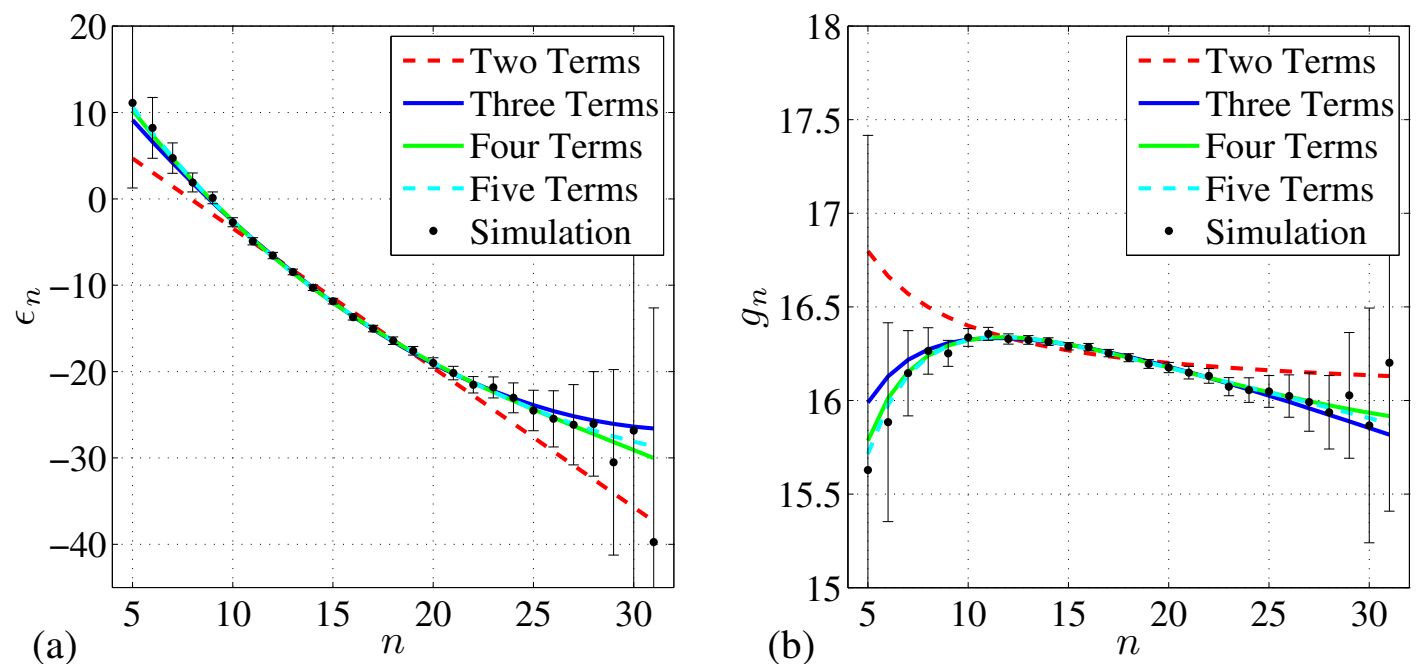

Figure 6. (a) $\epsilon_{n}$ and (b) $g_{n}$ as functions of $n$ for the three-dimensional PoissonVoronoi structure where $\mu_{2}$ is 11.197. Simulation results appear as black dots, while red, blue, green and cyan curves use values of $\epsilon_{n}$ given by least-squares fits of (37) with $c_{k}=0$ for $k$ greater than one, two, three and four, respectively. Fitting coefficients, given to the precision of the fitting procedure and ordered by increasing $k$, are -1.6152 for the red curves, -1.6014 and 0.046105 for the blue curves, $-1.5618,0.047520$ and -0.0011794 for the green curves, and $-1.5580,0.04481,-0.0013255$ and 0.000042989 for the cyan curves. Error bars indicate $95 \%$ confidence intervals on the mean.
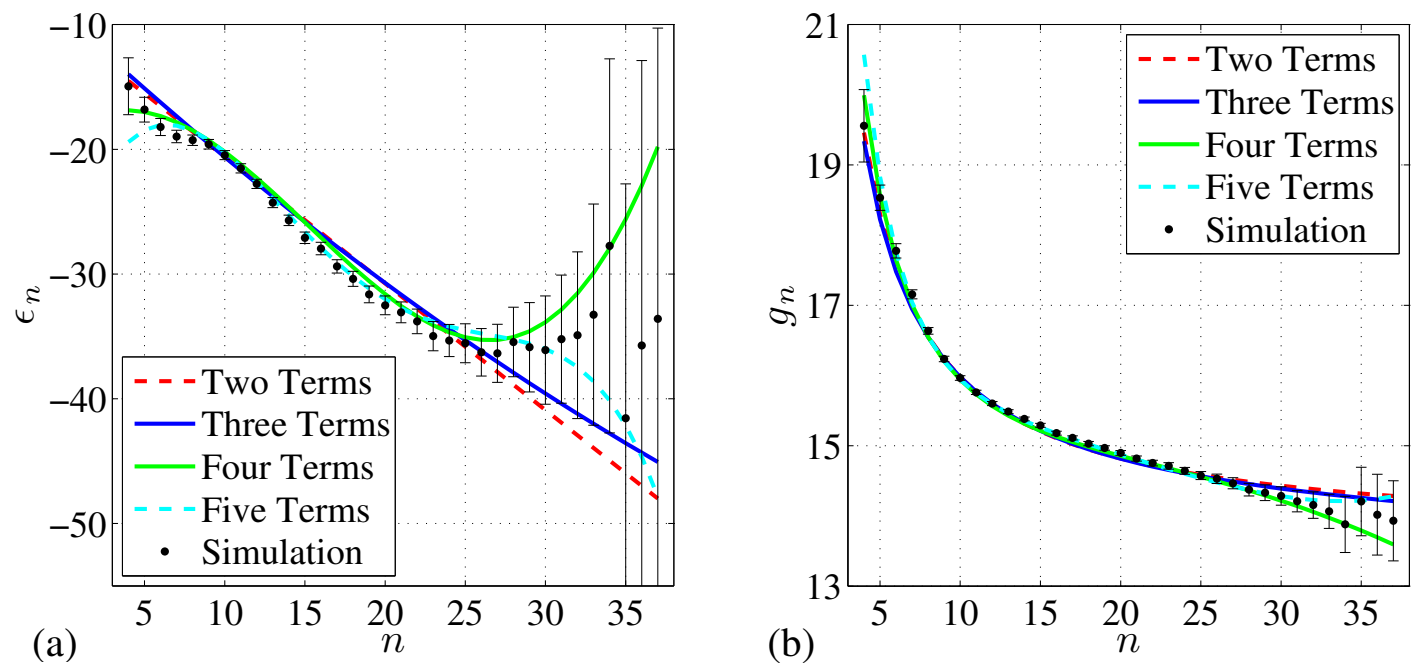

Figure 7. (a) $\epsilon_{n}$ and (b) $g_{n}$ as functions of $n$ for the three-dimensional steady state structure where $\mu_{2}$ is 22.125 . Simulation results appear as black dots, while red, blue, green and cyan curves use values of $\epsilon_{n}$ given by least-squares fits of (37) with $c_{k}=0$ for $k$ greater than one, two, three and four, respectively. Fitting coefficients, given to the precision of the fitting procedure and ordered by increasing $k$, are -1.01467 for the red curves, -1.02431 and 0.006095 for the blue curves, $-1.2008,-0.013849$ and 0.00317284 for the green curves, and $-1.3436,0.005082,0.00626411$ and -0.0002500819 for the cyan curves. Error bars indicate $95 \%$ confidence intervals on the mean. 